\title{
Sol-Gel Transition of Acid Silica Sols Produced by a Y-shaped Reactor
}

\author{
Saori KIKUCHI ${ }^{*}$, Takashi SAEKI ${ }^{*}$, Masahiro ISHIDA ${ }^{*}$, Kazuaki TABATA ${ }^{* *}$, and Kohzo OHTA ${ }^{* *}$ \\ "Graduate School of Science and Engineering, Yamaguchi University, \\ 2-16-1, Tokiwadai, Ube, 755-8611, Japan \\ ${ }^{* *}$ Tokuyama Corporation, 1-1, Mikage-cho, Shunan, 745-8648, Japan
}

(Received: May 17, 2010)

\begin{abstract}
We have developed a new production method for silica sols using a Y-shaped reactor. Diluted sodium silicate and sulfuric acid were forced to collide against each other at the intersection of the reactor. Silica sols with the appropriate viscosity produced by this method could be used as waste water treatment agents and retention aids for papermaking. In this study, the gelation process of silica sols was considered by dynamic viscoelastic measurements. Furthermore, we observed directly observed the gel surface using a scanning probe microscope (SPM). From the results, the onset time of gelling was strongly affected by both the concentration of $\mathrm{SiO}_{2}$ and the excessive rate of sulfuric acid. The gel surface structure produced with different conditions was also characterized. It was found that larger coalescence of silica particles presented in gels and the coalescence rate depended on the excessive rate of sulfuric acid.
\end{abstract}

Key Words: Silica / Sol-gel transition / SPM

\section{INTRODUCTION}

Silica particles have been receiving considerable attention in both scientific and industrial fields as particulate materials, hardeners, absorbing agents, agglomerate reagents, and so on. Numerous studies on silica have been conducted, and they were summarized by Iler in 1979. ${ }^{1)}$ In his book, typical solgel transitions for silica sols were explained in detail. Further studies concerned with silica sols prepared from silicon alkoxide, solvents and catalysts have recently been conducted.

Acid silica sols are produced with sodium silicate and sulfuric acid by their neutralization, as described in the following reaction formula;

$$
\mathrm{Na}_{2} \mathrm{O} \cdot \mathrm{nSiO}_{2}+\mathrm{H}_{2} \mathrm{SO}_{4} \rightarrow \mathrm{nSiO}_{2}+\mathrm{Na}_{2} \mathrm{SO}_{4}+\mathrm{H}_{2} \mathrm{O}
$$

This reaction is suitable for manufacturing silica sols by the comparatively simple operation with cheap raw materials. The reaction is performed by a batch vessel, in which sodium silicate solution is added to the vessel with the appropriate amount of sulfuric acid. During the addition, mild agitation is conducted for around one hour, however; it is impossible to produce homogeneous silica sols which have a solid

Phone/Fax: +81-836-85-9242, E-mail: saeki@yamaguchi-u.ac.jp concentration of more than $6 \mathrm{wt} \%$ due to localized gelling problem.

In our previous work, ${ }^{2)}$ the sol-gel transition of the acid silica sols was experimentally considered by using a rheometer, light scattering measurement, and electric conductivity measurement. In this study, a Y-shape reactor ${ }^{3)}$ was used to produce silica sols. The reactor had two inlets, in which dilute sodium silicate and sulfuric acid were supplied. Two solutions were forced to collide with each other at the intersection of the Y-shaped reactor. Using this method, we can produce homogeneous silica sols continuously and obtain silica sols with a concentration of up to $14-16 \mathrm{wt} \%$. The onset times of gelling were determined for several preparation conditions.

Since gel aging is caused by the formation of the silica particles network, it is necessary not only to measure the rheological properties during sol-gel transition, but also to analyze the structure of the formed gel surface directly to understand the mechanism of sol-gel transition.

Rheological studies on silica sols and their gel transition were conducted for that produced by the silicon alkoxide system. Ro reported ${ }^{4)}$ that prepared silica sols by silicon alkoxide initially behaved as Newtonian fluids. As time passed (and aging occurred), the viscosity gradually increased and 
displayed shear thinning property. Chen ${ }^{5)}$ and Pope ${ }^{6}$ measured both the viscosity and density of silica sols and confirmed that the ratios of components and the difference of catalysts affected the rates of gelation and the gel properties. Recently, viscoelastic measurements have been adopted to analyze the sol-gel transition. Takahashi et al. investigated the gel forming of polymer blends during aging. ${ }^{7)}$ The terminal behavior $\mathrm{G}^{\prime} \sim \omega^{2}$ and $\mathrm{G}^{\prime \prime} \sim \omega$ are exhibited for Newtonian fluids. ${ }^{8,9)}$ For sol-gel transition caused by percolation of particles, both $\mathrm{G}^{\prime}$ and $\mathrm{G}^{\prime \prime}$ are proportional to the frequency to the nth power at the gel point. ${ }^{10)}$

On the other hand, atomic force microscope (AFM) is frequently used to observe the surface structure of various materials. AFM can obtain surface images of soft materials such as polymer, gel and DNA by using the tapping mode. ${ }^{11,12)}$ The permeability of silica membranes was discussed from observation of their surface. ${ }^{13,14)}$ Scanning with the tapping mode of AFM was adopted, and can probe the surface without strong deformation compared to the contact mode. However, the observation of surface structure for soft materials has some difficulties due to the effect of between the cantilever and substance. Recently, scanning probe microscopy (SPM) has been widely used to characterize the nature of surfaces, such as viscoelasticity, adhesion or friction of materials. The relation phases of the cantilever oscillation with respect to the source driving the oscillation are imaged as the surface image. ${ }^{15-17)}$ Therefore, the technique is one of the best ways to observe composite surfaces of multiphase materials with different physical properties. ${ }^{15,18)}$ The silica particles in sols prepared from silicon alkoxide were observed by SPM. ${ }^{19)}$ The electric potential distribution maps obtained by SPM show significant interparticle property, especially in the case of smaller particles.

In this study, the sol-gel transition of acid silica sols produced with different excessive rate of sulfuric acid using a Y-shaped reactor was investigated. The sol-gel transition was observed by measurement of the viscoelasticity. Both dispersed particles in sols and the gel surface were observed to consider the transition process. The size and bonding array of silica particles provide crucial information needed to clarify the mechanism of the sol-gel transition, thus enabling improvement of its application in product development.

\section{EXPERIMENTAL}

\subsection{Materials and Sol Production}

The $\mathrm{SiO}_{2}$ concentration of sodium silicate solutions used in this experiment was $280-295 \mathrm{~g} / \mathrm{L}$, while the concentration of sulfuric acid solutions was 200-220 g/L. The molecular ratio of $\mathrm{Na}_{2} \mathrm{O}$ and $\mathrm{SiO}_{2}(=\mathrm{M} \cdot \mathrm{R})$ were between 2.9 and 3.2.

Fig. 1 shows the experimental apparatus for producing silica sols, which consisted of a sulfuric acid line (A) and a sodium silicate line (B). Each line was composed of a tank, a pump, and a flow meter. The flow rates of both sulfuric acid and sodium silicate were controlled by inverters installed in each pump (A or B). The flow rate of each solution was more than $10 \mathrm{~m} / \mathrm{sec}$. Both lines were connected to the inlets of the Y-shaped reactor. ${ }^{3)}$ The silica sols produced were obtained from the outlet of the reactor. The silica sols were produced with various flow rate conditions. Here, the concentration of $\mathrm{SiO}_{2}$ in a produced silica sol, $\mathrm{C}$, and the excess rate of sulfuric acid, k, were given by definitions (2) and (3).

$$
\begin{gathered}
C=C^{\prime} \times \frac{Q_{2}}{Q_{1}+Q_{2}} \\
k=\frac{C_{1} \times Q_{1} \times M_{2}}{C_{2} \times Q_{2} \times M_{1}}
\end{gathered}
$$

where $\mathrm{C}^{\prime}, \mathrm{Q}_{1}$, and $\mathrm{Q}_{2}$ indicate the concentration of $\mathrm{SiO}_{2}$ in sodium silicate solution, the flow rate of sulfuric acid solution, and that of sodium silicate solution, respectively. $\mathrm{C}_{1}$ and $\mathrm{C}_{2}$ indicate the concentration of sulfuric acid solution, and that of sodium silicate solution, respectively.

\subsection{Rheological Measurement}

The rheological properties of silica sols were measured using a coaxial-cylinder rotating rheometer (A-300, Elquest Co. Ltd., Japan). The steady shear viscosity at the shear rate of $20 \mathrm{~s}^{-1}$ and the viscoelasticity at the frequency of $1 \mathrm{~Hz}$ and the strain of 0.1 were measured.

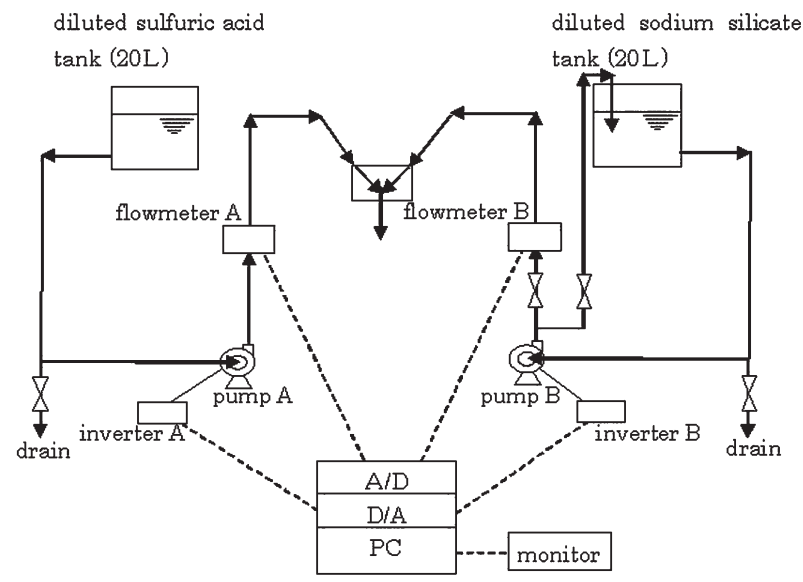

Fig. 1. Experimental apparatus. 


\subsection{Visualization Experiments}

Visualization experiments were performed for samples obtained both before and after the gel point. The silica particles in sols were observed by using the freeze-replica method (FRM). A silica sol was obtained just before its gel point and was diluted by adding water to the $\mathrm{SiO}_{2}$ concentration of $0.02 \%$. The sol was dropped on the liquid nitrogen slush, and the frozen silica sol was cut to make a flat surface. Carbon paper was placed on the surface of the frozen sol and the ice was removed by putting into water. The silica particles remaining on the carbon paper were observed by a TEM.

On the other hand, silica gel was obtained after its gel point. Then, a piece of the gel was laid flat to make the surface of the sample stage of SPM (SPM-9600 Shimazu Co. Ltd), the gel and sample stage were thus parallel. SPM images of the gel surface were obtained by the tapping mode. The moisture on the silica gel surface was removed by using tissue. The phase shifts of the cantilever affected by the local viscoelasticity of the gel surface were converted to SPM images.

\section{RESULTS AND DISCUSSION}

\subsection{Rheological Measurement}

The storage modulus, G', loss modulus, G", and $\delta$ of silica sols during gel aging are shown in Fig. 2 In the early stage of gel aging, $\mathrm{G}^{\prime \prime}$ was greater than $\mathrm{G}^{\prime}$ and both values gradually increased. At the sudden increase of both values in Fig. 2, the magnitude relation of them reversed, and the sample was thus expected to tend to show the elastic property rather than the viscous property. The intersection of $\mathrm{G}^{\prime}$ and $\mathrm{G}^{\prime \prime}$ around their rapid jump is defined as a gel point, when the value of $\delta$, the $\arctan$ of $G^{\prime \prime} / G^{\prime}$, is $\pi / 4$. Around the gel point in this figure, the value of $\delta$ rapidly decreased from 1.4 to 0 , and then the values of $\mathrm{G}^{\prime}$ and $\mathrm{G}^{\prime \prime}$ continued to increased due to rigid gelation.

The steady shear viscosity and $\delta$ of silica sols during gel aging are shown in Fig. 3. In our previous work ${ }^{2}$, the time at the onset of the sudden increase in the steady shear viscosity was considered to coincide with the onset of the sol-gel transition. The onset time is determined as indicated A in Fig. 3 , while the gel point, determined by $\delta$ is indicated as B. Fig. 4 shows both $\mathrm{G}^{\prime}$ and $\mathrm{G}^{\prime \prime}$ of a silica sol as a function of frequency around the gel point. Since the silica sol still had fluidity at $\mathrm{t}=34860 \mathrm{~s}$, both $\mathrm{G}^{\prime}$ and $\mathrm{G}^{\prime \prime}$ depend on the frequency at which $\mathrm{G}^{\prime}$ is proportional to $\omega^{1.7}$ and $\mathrm{G}^{\prime \prime}$ is proportional to $\omega$. For typical polymer solutions, $G^{\prime}$ and $G^{\prime \prime}$ exhibit a low-frequency terminal behavior. For Newtonian fluids, $G^{\prime}$ is proportional to $\omega^{2}$ and $G^{\prime \prime}$ is proportional to $\omega .^{7-9)}$ The dependence of $G^{\prime}$ on frequency became lower with increasing aging time. At the gel point of $\mathrm{t}=36780 \mathrm{~s}$, both $\mathrm{G}^{\prime}$ and $\mathrm{G}^{\prime \prime}$ are proportional to $\omega^{\mathrm{n}}$, in which $\mathrm{n}$ is determined as 0.673 at the gel point. Here, it is reported that $n=2 / 3$ for the sol-gel transition based on the percolation theory. ${ }^{10,20)}$ Therefore, the mechanism of the solgel transition for acid silica sols is expected to be the same as that described in the percolation theory. After the gel point, the dependence of $\mathrm{G}^{\prime}$ on the frequency was not exhibited due to

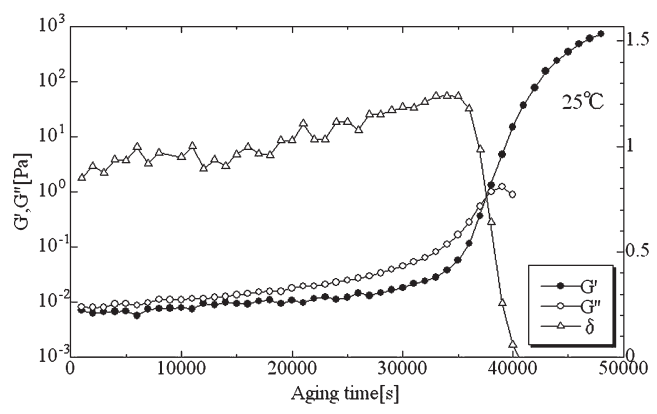

Fig. 2. Viscoelastic property during gel aging of the silica sol. (concentration of $\mathrm{SiO}_{2}=13 \mathrm{~g} / 100 \mathrm{ml}$, excessive rate of sulfuric acid $=1.15)$

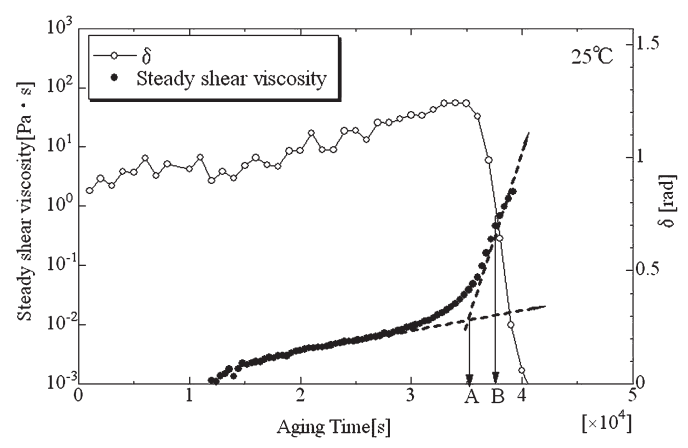

Fig. 3. Observations of gel aging in terms of viscosity and viscoelasticity. (concentration of $\mathrm{SiO}_{2}=13 \mathrm{~g} / 100 \mathrm{ml}$, excessive rate of sulfuric acid $=1.15$ )

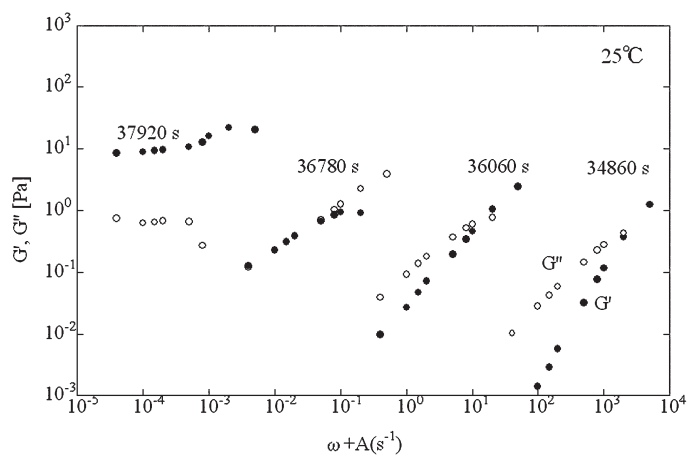

Fig. 4. Dependence of storage modulus $\mathrm{G}^{\prime}$ and loss modulus $\mathrm{G}^{\prime \prime}$ on the angular frequency measured for silica sols (gels) at various aging time. (concentration of $\mathrm{SiO}_{2}=13 \mathrm{~g} / 100 \mathrm{ml}$, excessive rate of sulfuric acid $=1.15$ )

* In Fig.4, the frequency values were shift for some value (A). 
the steady rigidity of the silica gel. The rigidity of silica gels continued to increase after gelling.

Fig. 5 shows the value of $\delta$ during the aging for silica sols produced with different excessive rates of sulfuric acid and concentrations of $\mathrm{SiO}_{2}$. The result showed that if the concentration of $\mathrm{SiO}_{2}$ increased or the excessive rate of sulfuric acid increased, the gel aging time became shorter.

For polymerization of silica, the coordinated number of $\mathrm{Si}$ which is included in mono silicic acid changes from 4 to 6 . Below $\mathrm{pH} 2, \mathrm{H}^{+}$ions form an active cationic complex which is consisted of a three-silicon shown in Fig. 6. The forming rate of the complex increases with increasing the concentration of $\mathrm{H}+$ ions. The complexes continue to link by Si-O-Si bonding to form the three dimensional molecular structure so called silica particles. Since silica particles with weak charge collide to bond irreversibly each other, the sol is in the metastable condition. The rate of gelation increases in proportional to the concentration of $\mathrm{H}^{+}$ions. ${ }^{1)}$

\subsection{Visualization of Gel Structure Formed by Silica Particles}

It is expected that acid silica sols gradually increase in viscosity due to the growth and bonding of silica particles. Silica particles in a sol were observed by FRM and TEM and

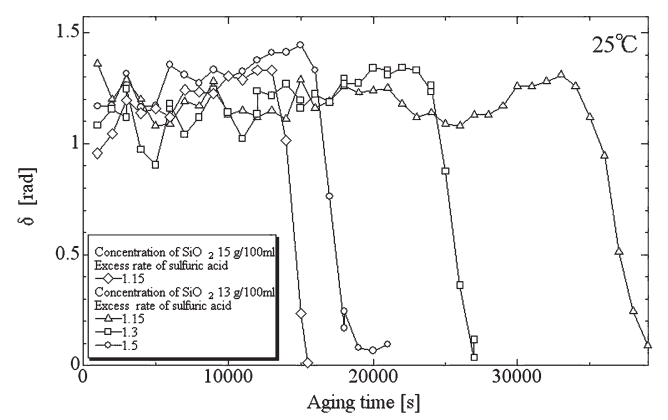

Fig. 5. The value of $\delta$ for silica sols produced by various conditions during aging measured by oscillation examination. (A-300)

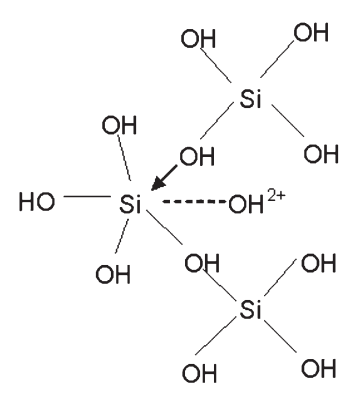

Fig. 6. Active cationic complex consisted of three monosilicic acid. ${ }^{1)}$ the obtained image is shown in Fig. 7. Mono dispersed silica particles with a diameter of $1 \sim$ several nm were observed. It was found that most silica particles existed without long linking.

Fig. 8 shows the surface image of the silica gel just after gelling as observed by the SPM. The color histogram in this image shows the rigidity level and distribution; the white areas indicate the rigid portions, while the dark areas show the soft portions compared with the surroundings. Here, the concentration of $\mathrm{SiO}_{2}$ of the sol was $13 \mathrm{~g} / 100 \mathrm{ml}$. Since the silica particles and their network on the probed surface were likely rigid compared with the water portion of the sol, the color bar was shifted by taking into consideration the $\mathrm{SiO}_{2}$ volumetric percent, as shown in Fig. 9(a). With the same modification, Figs. 9(b) and 9(c) show the surface of the same silica gel observed at 17 hours and 45 hours after gelling, respectively. It was found that the silica particles increased in diameter with the time increase, and a significant amount of partial coalescence was confirmed 45 hours after gelling. Iler $^{1)}$ introduced an electron microscopic study of silica gel,

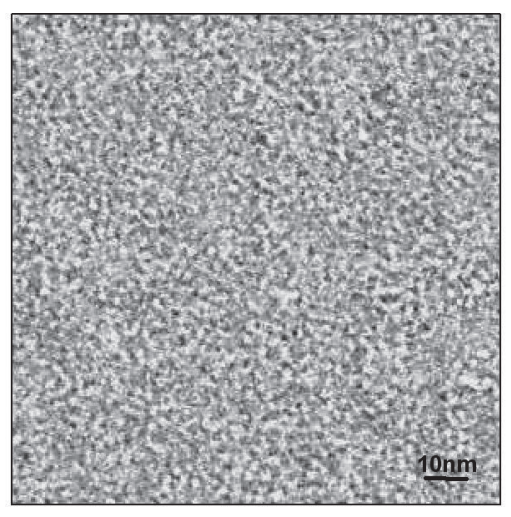

Fig. 7. The silica particles in sols observed by the TEM. (concentration of $\mathrm{SiO}_{2}=0.02 \%$ )

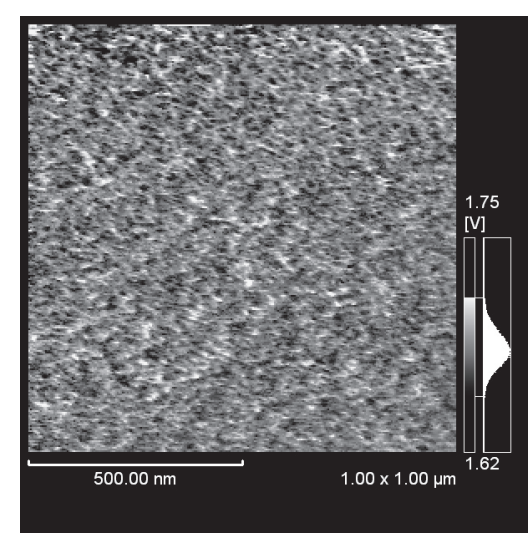

Fig. 8. SPM image of the silica gel just after gelling. (concentration of $\mathrm{SiO}_{2}=13 \%$, excessive rate of sulfuric acid $=1.5$ ) 
and mentioned that chains of particles can develop into rods or fibers until they essentially lose their particulate identity. Iler also stressed that the coalescences, formation of chains of particles, and networks were also observed for the silica gel by the SPM.

Fig. 10 shows the surface of the silica gel produced with a lower excessive rate of sulfuric acid. Since the size and distribution of silica particles in Fig. 10(a) are almost the same level as in Fig. 9(a), it was found that the diameter size of the silica particles just after gelling was not affected by the excessive rate of sulfuric acid. The growth and coalescence of silica particles was also confirmed in Fig. 10, but the degree of coalescence was inferior to that in Fig. 9. As shown in Fig. 7, the TEM image obtained for silica sols before the gel point showed that silica particles dispersed homogeneously, while the SPM images show the bonding and percolating of silica particles due to the progress in time and finally the form of the gel structure.

Although similar SPM images were observed just after gelling, the onset time of gelling depended on the excessive rate of sulfuric acid. Since the concentration of particles is the same according to the reaction indicated in Eq.(1), the surface activity is expected to relate to the forming of networks built by silica particles. It should be pointed out that the coalescence rate of silica particles increased with decreasing onset time of sol gelling.

The gel aging model for acid silica sols is summarized in Fig.11. Mono dispersed silica particles exist in the sol just after the neutralized reaction [Fig. 11(a)]. The viscosity of silica sols gradually increase due to the particles growing and bonding with each other [Fig. 11(b) and (c)]. The silica particles bond to each other to reach the gel point, the socalled percolated state [Fig. 11(d)]. After the gel point, free silica particles deposit on the network of silica particles [Fig. 11(e)] and the coalescences of silica particles progress [Fig. 11(f)]. From the result in this study, it was indicated that the coalescence rate of silica particles depends significantly on the excessive rate of sulfuric acid.

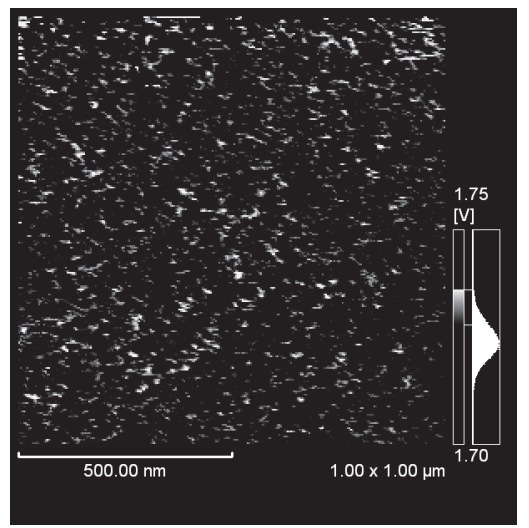

(a) Just after gelling

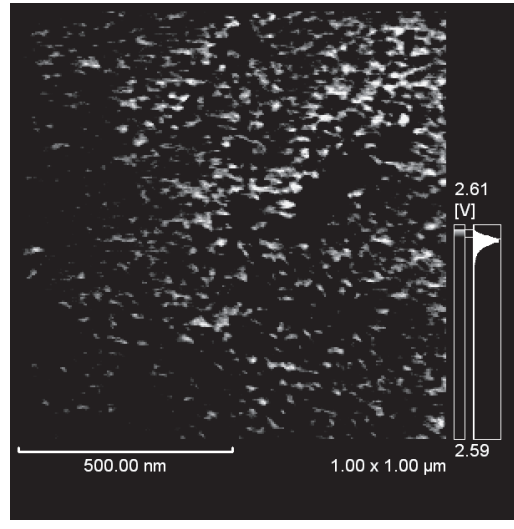

(b) 17 hours passed after gelling

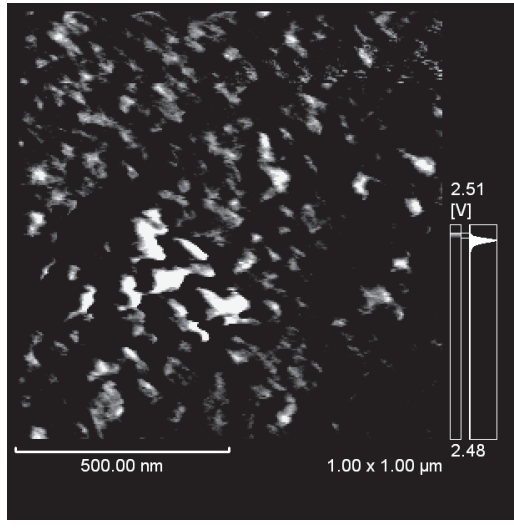

(c) 45 hours passed after gelling

Fig. 9. Modified SPM images of silica gels. (concentration of $\mathrm{SiO}_{2}=13 \%$, excessive rate of sulfuric acid =1.5)

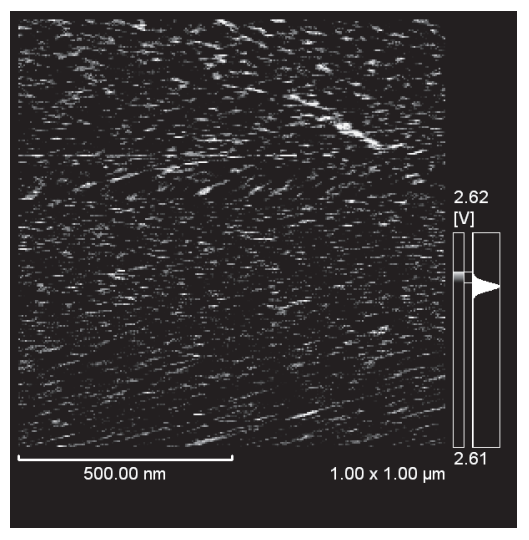

(a) Just after gelling

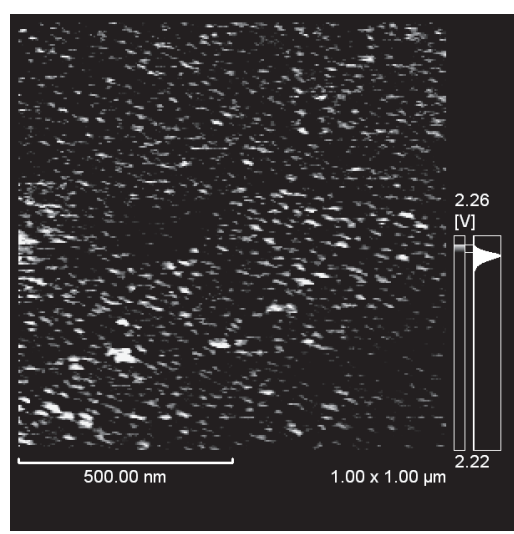

(b) 17 hours passed after gelling

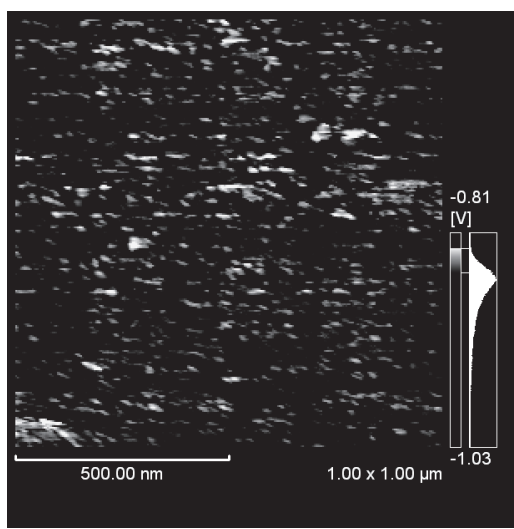

(c) 45 hours passed after gelling

Fig. 10. Modified SPM images of silica gels surfaces. (concentration of $\mathrm{SiO}_{2}=13 \%$, excessive rate of sulfuric acid $=1.15$ ) 


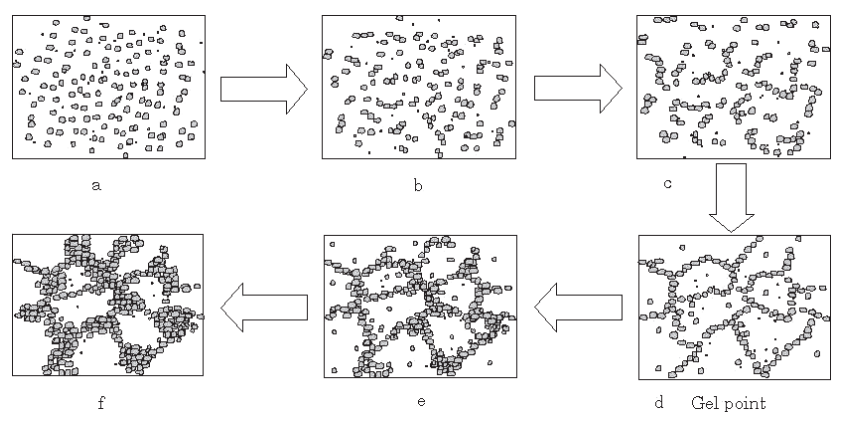

Fig. 11. Gel aging model of silica particles for sol-gel transition of silica sols. The explanation of each part is given in the next.

\section{CONCLUSION}

Sol-gel transitions of acid silica sols produced by a Y-shaped reactor with various conditions were observed in terms of their rheological characteristics. From the viscoelasticity measurement, the sol-gel transition of silica sols were characterized as the gel formation based on the percolation theory. The preparation conditions for silica sols affected the onset time of gelling. The gel structures were observed by SPM, and it was confirmed that the particles bonded and coalesced to form gel. Although the onset times of gelling were different, the SPM images obtained just after gelling shows a similar structure. The surface activity was expected to relate to the formation of networks built by the silica particles.

\section{NOMENCLATURE}

$$
\begin{array}{ll}
\mathrm{C}, \mathrm{C}^{\prime} & =\text { concentration } \\
\mathrm{k} & =\text { excess rate of sulfuric acid } \\
\mathrm{M} & =\text { molecular weight } \\
\mathrm{Q} & =\text { flow rate } \\
\mathrm{G}^{\prime} & =\text { storage modulus } \\
\mathrm{G}^{\prime \prime} & =\text { loss modulus } \\
\delta & =\text { phase difference }
\end{array}
$$

$<$ subscripts $>$

$$
1=\text { sulfuric acid }
$$$$
2=\text { sodium silicate }
$$

\section{REFERENCES}

1) Iler KR, The Chemistry of Silica, in Solubility, Polymerization, Colloid and Surface Properties and Biochemistry of Silica, John Wiley and Sons Inc. (1979).

2) Kikuchi S,Saeki T, Tabata K, Ohta K, e-J Surf Sci Nanotech, 7, 1-4 (2009).

3) Koga Y, Kosuga M, Proc of TAPPI Int Conf on Nanotechnology, 23 (2006).

4) Ro JC, Chung IJ, J Non-Cryst Solid, 126, 259-266 (1990).

5) Chen KC, Tuchiya T, Mackenzie JD, J Non-Cryst Solid, 81, 227-237 (1986).

6) Pope EJA, Mackenzie JD, J Non-Cryst Solid, 87, 185-198 (1986).

7) Takahashi H, Ishimuro Y, Watanabe H, Nihon Reoroji Gakkaishi, 35 (4) 191-198 (2007).

8) Kobayashi K, Huang CI, Lodg TP, Macromolecules, 32, 7070-7077 (1999).

9) Zhang Y, Xu X, Zhang L, Carbohydrate Poly, 73, 26-34 (2008).

10) Takenaka M, Kobayashi T, Saijo K, Tanaka H, Iwase $N$, Hashimoto T, Takahashi M, J Chem Phys, 121, 7 (2004).

11) Hobbs JK, Mullin N, Weber CHM, Farrance OE, Vasilev C, Materials, 12, 7-8 (2009).

12) Tripathi A, Kumar A, Singh F, Kabiraj D, Avasthi DK, Pivin JC, Nucl Instr And Meth B, 236, 186-194 (2005).

13) Oyama ST, Leed D, Sugiyama S, Fukui K, Iwasawa $Y, J$ Mater Sci, 36, 5213-5217 (2001).

14) Fujii T, Yano T, Nakamura K, Miyawaki O, J Mater Sci, 187, 171-180 (2001).

15) Valadares LF, Linares EM, Braganca FC, Glembeck F, $J$ Phys Chem C 112, 8534-8544, (2008).

16) Greene ME, Kinser CR, Kramer DE, Pingree LSC, Micro research and Tech, 64, 415-434 (2004).

17) Bhushan B, Wear, 251, 1105-1123 (2001)

18) Nakamura H, Mitsuoka $T$, Ishii M, J Appli Poly Scie, 102, 2308-2314 (2006)

19) Costa CAR, Leite CAP, Souza EF, Galembeck F, Langmuir, 17, 189-194 (2001).

20) Chambon F, Winter HH, Polym Bull 13, 499-503 (1985). 